\title{
Energy trading systems on blockchain networks
}

\section{Jae Geun Song and Ju Wook Jang*}

Department of Electronic Engineering, Sogang University, Seoul, South Korea
Received: 26 June, 2020

Accepted: 04 July, 2020

Published: 07 July, 2020

*Corresponding author: Ju Wook Jang, Department of Electronic Engineering, Sogang University, Seoul, South Korea, Tel: +82-10-3744-8472; Fax: +82-2-3272-3220; E-mail: jjang@sogang.ac.kr

Keywords: Blockchain; Energy trading; P2P trading https://www. peertechz.com

\section{Abstract}

Traditional energy trading system has some weakness in terms of network attack. So blockchain is considered for P2P energy trading systems to evade network attacks. We consider three energy trading systems on blockchain which are centralized energy trading system with one producer, $\mathrm{P} 2 \mathrm{P}$ energy trading system with $\mathrm{DSO}$ and P2P energy trading system with smart contract. And we compared P2P energy trading systems on running in terms of transaction fees. By using blockchain, users can reduce transaction fees then make more benefits.

\section{Abbreviations}

DSO: Distributed System Operator; IEA: International Energy Agency

\section{Introduction}

Production of renewable energy such as solar energy is increased from all over the world. If an energy producer produces too much energy for consumption, renewable energy can be wasted. Then the producer can make decision to sell surplus energy for increasing energy efficiency. However, there are some possible network attack when trading surplus energy on traditional centralized network $[1,2]$. Following threats are example of possible attack.

IP spoofing: If a malicious node attacks the NRS (Naming Resolution System) server and forges the IP address of a specific node, the user cannot communicate with the desired node because of the wrong IP address [3].

Sybil attack: A malicious node masquerades as other node(s) [4].

Single point of failure: In a centralized network, authentication and trading activities will be disabled in the event of a failure or attack on key nodes [5].

Lack of privacy and anonymity: A node may reveal patterns of an agent's energy generation and predict the agent's daily activities [6].
Each network attack can exploit transaction data which makes fundamental loss to energy trading system. Blockchain can be a solution to evade network attacks. Different from the centralized digital ledger approaches, blockchain does not rely on centralized authorities to store transaction data. Instead, data blocks are recorded and shared by blockchain users over the whole blockchain network. And blockchain refers to a distributed ledger which is shared by all the nodes participating in a $\mathrm{P} 2 \mathrm{P}$ network. The distributed ledger records transactions with a chain of hashes to ensure its integrity [7]. As a result, malicious attacker can't success their attacks in blockchain networks. For example, the balance attack against proof of work in R3 testbed was failed [8]. Because of these advantages, many developers wanted to create a decentralized energy trading architecture using blockchain and we also consider energy trading system on blockchain. There has been much effort to utilize the capability of blockchain for energy trading in local neighborhood such as microgrid [9]. The units of generated energy are recorded in a block and the producers may sell extra energy to participants of the network. This enables energy producers and buyers trade energy without any intermediary parties involved. A few researchers on P2P energy trading show that blockchain based intermediary-free energy trading is not only possible but also beneficial to all the producers and buyers.

\section{Materials and methods}

We regard the energy trading systems on blockchain network based on the previous works [10]. So we can see that 
the energy trading systems are depending on a structure of the power transmission and distribution grid $[11,12]$. As a result, we consider the following three energy trading systems according to the structure of the power grid.

(Figure 1) shows Centralized energy trading system with one producer which is similar to existing centralized energy transmission/distribution system without blockchain. This system is suitable when a supply of energy can be provided stably. In this system the energy producer can modulate energy price flexibly, energy buyers should purchase an energy for a fixed price determined by the energy producer. Because energy producer can reduce the network maintenance and security costs rather than traditional system, if the energy producer supplies energy as a lower price, energy buyer can purchase an energy as a lower price than a system without blockchain.

(Figure 2) shows P2P Energy Trading System with DSO which is a system consists of DSO and multiple energy producers and buyers. This system is suitable when an energy producer wants to sell an energy but there is no direct transmission and distribution grid with a buyer. In this case, the energy producer sells the energy to the buyer through transmitting the energy to the DSO and DSO transmits the energy to the buyer. DSO can make a profit by receiving fees for using power grid or trading. Energy producers and buyers can make a profit by trading at a lower price than existing energy transaction prices.

(Figure 3) shows P2P energy trading system with smart contract which is a system consists of energy producers and buyers with smart contract. This system is suitable when energy buyers and producers can directly transmit/distribute. In this system, producers and buyers use smart contract like DSO to increase reliability of transactions. Both buyer and producer can make periodically transaction by using smart contract. Then both buyers and producers can maximize their profits by reducing transaction fees with minimal intervention.

\section{Results}

Changing the existing system to blockchain-based system like A, B, C can reduce transaction fees. (Figure 4) shows

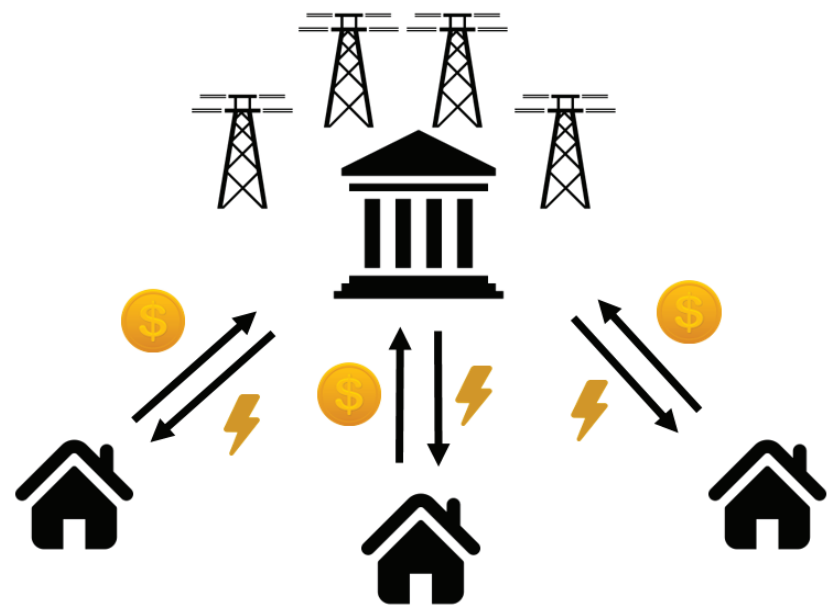

Figure 1: Centralized energy trading system with on producer.



Figure 2: P2P Energy trading system with DSO.



Figure 3: P2P Energy trading system with smart contract.

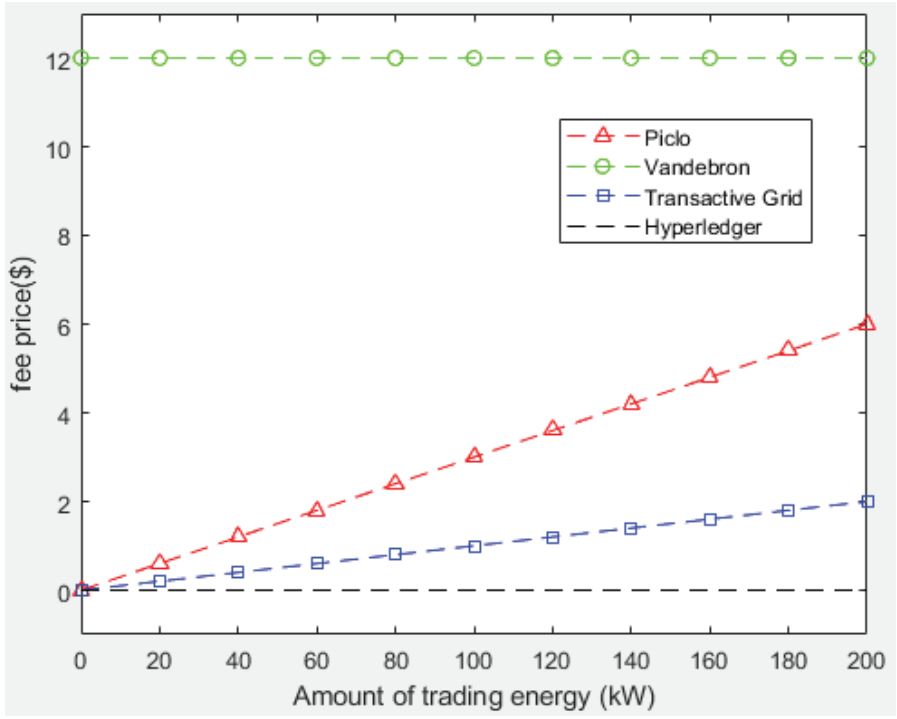

Figure 4: Transaction fees for each Energy trading platform.

Citation: Song JG, Jang JW (2020) Energy trading systems on blockchain networks. Trends Comput Sci Inf Technol 5(1): 018-022. DOI: https://dx.doi.org/10.17352/tcsit.000014 
transaction fees for each energy trading platform. We simulate under energy price is \$0.2 from IEA for Piclo and 1 transaction per $1 \mathrm{~kW}$ for Transactive Grid [9]. Piclo and Vandebron are not blockchain-based each charges $15 \sim 19 \%$ of energy price and $\$ 12$ as a month [13]. However, Transactive Grid is Ethereum blockchain-based energy trading platform which is a P2P energy trading system with smart contract. In Transactive Grid, participants pay \$0.01 per transaction but it is lower than Piclo and Vandebron. Even more, hyperledger has no transaction fees when trading energy because hyperledger is based on permissioned blockchain [14].

\section{Conclusion}

Changing the existing energy trading system into a blockchain based energy trading system brings lower fees than previous. And we propose three blockchain based energy trading system for each power grid structure. Each system's benefit is depending on blockchain structure. For example, ethereum has higher transaction fees than hyperledger but it is more easily applicable. However, there are some threats to blockchain systems. 51\% vulnerability, private key security, criminal smart contracts, vulnerabilities in smart contract and transaction privacy leakage are possible problems [15]. So, we still need to compare the costs used to build these systems with the expected benefits.

\section{Conflict of interest}

Gap of cost used to build system and expected benefits can be conflict point. And in centralized energy trading system with one producer, producer doesn't have to discount energy price. Therefore, buyers don't have any benefits while producer makes more revenue.

\section{Acknowledgement}

This work was supported by the "Development of a service platform for trading and sharing excess electricity on residential houses" the Korea Institute of Energy Technology Evaluation and Planning(KETEP) granted financial resource from the Ministry of Trade, Industry \& Energy, Republic of Korea (No. 20191210301820).

\section{References}

1. Nurzhan Z, Davor S (2018) Security and Privacy in Decentralized Energy Trading Through Multi-Signatures, Blockchain and Anonymous Messaging Streams. IEEE Transaction on Dependable and Secure Computing 15. Link: https://bit.ly/2NVJzEt

2. Gary SA (2009) Single Point of Failure: The 10 essential laws of supply chain risk management. John Wiley and Sons. Link: https://bit.ly/31MOAHq

3. Matthew T (2003) IP spoofing: an introduction.

4. Douceur JR (2002) The sybil attack. International Workshop on Peer-to-Peer Systems 251-260. Link: https://bit.ly/31MOUpC

5. Alynch, Gary S (2009) Single Point of Failure: The 10 essential laws of supply chain risk management. John Wiley and Sons 320. Link: https://bit.ly/31MOAHq

6. Aitzhan NZ, Svetinovic D (2018) Security and Privacy in Decentralized Energy Trading Through Multi-Signatures, Blockchain and Anonymous Messaging Streams. IEEE Transactions on Dependable and Secure Computing 15. Link: https://bit.ly/2NVJzEt

7. Zikratov I, Kuzmin A, Akimenko V, Niculichev V, Yalansky L (2017) Ensuring data integrity using blockchain technology. Conference of Open Innovations Association (FRUCT) Link: https://bit.ly/2ZHi9aQ

8. Natoli C, Gramoli V (2016) The Balance Attack Against Proof-Of-Work Blockchains: The R3 Testbed as an Example. Computer Science. Link: https://bit.ly/2NWoE3R

9. Esther M, Johannes G, Kerstin R, Scott K, Lawrence O, et al. (2018) Designing microgrid energy markets: A case study: The Brooklyn Microgrid. Applied Energy 210: 870-880. Link: https://bit.ly/3dZQYgp

10. Johnston J (2017) Peer-to-Peer Energy Matching: Transparency, Choice, and Locational Grid Pricing. Innovation and Disruption at the Grid's Edge How Distributed Energy Resources are Disrupting the Utility Business Model 319330

11. Mylrea M, Gourisetti SNG (2017) Blockchain for smart grid resilience. Exchanging distributed energy at speed, scale and security. 18-23. Link: https://bit.ly/200LR5g

12. Mylrea M, Gourisetti SNG (2017) Blockchain: A path to grid modernization and cyber resiliency. North American Power Symposium (NAPS). Link: https://bit.ly/2AwpPE7

13. Zhang C, Wu J, Zhou Y, Cheng M, Long C (2018) Peer-to-Peer energy trading in a Microgrid. Applied Energy 220: 1-12. Link: https://bit.ly/2VN6gPy

14. Androulaki E, Barger A, Bortnikov V, Cachin C, Christidis K, et al. (2018) Hyperledger fabric: a distributed operating system for permissioned blockchains, EuroSys '18: Proceedings of the Thirteenth EuroSys Conference 1-15. Link: https://bit.ly/31ULYXU

15. Li X, Jiang P, Chen T, Luo X, Wen Q (2017) A survey on the Security of Blockchain Systems. Future Generation Computer Systems 107: 841-853. Link: https://bit.ly/2C6UTe7

Discover a bigger Impact and Visibility of your article publication with

Peertechz Publications

Highlights

* Signatory publisher of ORCID

* Signatory Publisher of DORA (San Francisco Declaration on Research Assessment)

* Articles archived in worlds' renowned service providers such as Portico, CNKI, AGRIS, TDNet, Base (Bielefeld University Library), CrossRef, Scilit, J-Gate etc.

* Journals indexed in ICMJE, SHERPA/ROMEO, Google Scholar etc.

* OAI-PMH (Open Archives Initiative Protocol for Metadata Harvesting)

- Dedicated Editorial Board for every journal

* Accurate and rapid peer-review process

* Increased citations of published articles through promotions

* Reduced timeline for article publication

Submit your articles and experience a new surge in publication services (https://www.peertechz.com/submission).

Copyright: @ 2020 Song JG, et al. This is an open-access article distributed under the terms of the Creative Commons Attribution License, which permits unrestricted use, distribution, and reproduction in any medium, provided the original author and source are credited. 
